\title{
BALANCED HOWELL ROTATIONS OF THE TWIN PRIME POWER TYPE
}

BY

DING-ZHU DU AND F. K. HWANG

\begin{abstract}
We prove by construction that a balanced Howell rotation for $n$ players always exists if $n=p^{r} q^{s}$ where $p$ and $q \neq 3$ are primes and $q^{s}=p^{r}+2$. This generalizes a much weaker previous result. The construction uses properties of a Galois domain which is a direct sum of two Galois fields.
\end{abstract}

1. Introduction. A balanced Howell rotation for $n=2 k$ players, denoted by $\operatorname{BHR}(n)$, consists of a set of $n$ players (denoted by $\infty, 0,1, \ldots, n-2$ ) and a set of $n-1$ boards (denoted by $0,1, \ldots, n-2$ ). For each board $i$ the $n$ players are divided into $k$ ordered pairs $\left(a_{i j}, b_{i j}\right), j=1, \ldots, k$, where $a_{i j}$ and $b_{i j}$ are said to oppose each other on board $i$, and $a_{i j}$ and each of $a_{i j^{\prime}}, j^{\prime} \neq j$, are said to compete with each other on board $i$. The partitions on the $n-1$ boards together must also satisfy the following two conditions.

(i) Each player opposes every other player exactly once.

(ii) Each player competes with every other player exactly $k-1$ times.

A $\operatorname{BHR}(n)$ can also be represented by an $(n-1) \times n$ array $A=\left(a_{i j}\right)$ where the rows are boards and the columns are players. Define $a_{i j}=k$ if $(j, k)$ is an opposing pair for board $i$ and define $a_{i j}=-k$ if $(k, j)$ is such a pair. Let $A^{*}$ be obtained from $A$ by adding a row $\infty$ such that $a_{\infty j}=j$. Then the signs in $A^{*}$ constitute a Hadamard matrix, and the numbers in $A^{*}$ constitute a latin square $L=\left(l_{i j}\right)$ with the property $l_{i j}=k \Rightarrow l_{i k}=j$ (called a tournament latin square). Of course, superimposing a Hadamard matrix on a tournament latin square does not automatically generate a $\operatorname{BHR}(n)$ unless for each row $i \neq \infty$, the signs of $a_{i j}=k$ and $a_{i k}=j$ are different for all $j$.

Direct constructions for $\operatorname{BHR}(n)$ 's have been given mostly when $n$ is related to a prime power, for example,

1. $n=P+1$ where $P=4 k+3$ is a prime power, $k \geqslant 1[1,5]$.

2. $n=2(P+1)$ where $P=2^{m} k+1$ is a prime power, $m \geqslant 1, k \geqslant 1$ and $k$ is odd $[2,4,6]$.

In [3], an attempt was made to construct $\operatorname{BHR}(n)$ 's when $n$ is related to a product of two prime powers differing by 2 (called twin prime powers). More specifically, it was proved (where $\mathrm{GF}^{*}(P)$ is the multiplicative group of $\mathrm{GF}(P)$ ) that

Received by the editors January 23, 1981.

1980 Mathematics Subject Classification. Primary 05B15; Secondary 05B10, 12C20.

1982 American Mathematical Society $0002-9947 / 82 / 0000-1053 / \$ 03.00$ 
Theorem 1 [3]. $A$ BHR $(n)$ exists if

(i) $n-1=P Q$ where $P$ and $Q$ are twin prime powers, and

(ii) there exist generators $x$ of $\mathrm{GF}^{*}(P)$ and $y$ of $\mathrm{GF}^{*}(Q)$ with $x^{a} \equiv 2(\bmod P)$, $P-2 \geqslant a \geqslant 0, y^{b} \equiv 2(\bmod Q), Q-2 \geqslant b \geqslant 0$, such that one of the following three cases holds: $b=a+1,(P-1) / 2 \geqslant b=a \geqslant 0$, and $P-2 \geqslant b-2 \geqslant(P+1) / 2$.

In this paper we look again into the twin prime power case and prove a much stronger result.

THEOREM 2. $A \operatorname{BHR}(n)$ exists if $n-1=P Q=p^{r} q^{s}$ where $P$ and $Q$ are twin prime powers, $P<Q$ and $q \neq 3$.

2. Some preliminary results. Let $x$ and $y$ generate $\operatorname{GF}^{*}\left(p^{r}\right)$ and $\operatorname{GF}^{*}\left(q^{s}\right)$, respectively. Let $G$ be the Galois domain (see [7]) $G=\mathrm{GF}\left(p^{r}\right) \oplus \mathrm{GF}\left(q^{s}\right)$ (direct sum), and let $U=\left\{(u, 0): u \in \mathrm{GF}\left(p^{r}\right)\right\}, V=\left\{(0, v): v \in \mathrm{GF}\left(q^{s}\right)\right\}$. Define $d=$ $(P-1)(Q-1) / 2$. The two cyclotomic classes in $G$ are

$$
\begin{aligned}
& C_{0}=\left\{\left(x^{i}, y^{i}\right), i=0,1, \ldots, d-1\right\}=\left\{\left(x^{i}, y^{j}\right), i=j(\bmod 2)\right\}, \\
& C_{1}=\left\{\left(-x^{i},-y^{i}\right), i=0,1, \ldots, d-1\right\}=\left\{\left(x^{i}, y^{j}\right), i \neq j(\bmod 2)\right\} .
\end{aligned}
$$

It is well known [7] that $C_{0}+U$ forms a difference set. Therefore $C_{1}+V-\{0\}$ is also a difference set.

Let the $n$ players be denoted by the elements in $G \cup\{\infty\}$. Suppose we can partition the $n$ players into $n / 2$ pairs $a_{i}$ vs. $b_{i}, i=1,2, \ldots, n / 2$, which meet the following two requirements.

(R1) $\pm\left(a_{i}-a_{j}\right)$ over all $i$, except the pair involving $\infty$, runs through the set of nonzero elements of $G$.

(R2) $\pm\left(a_{i}-a_{j}\right), \pm\left(b_{i}-b_{j}\right)$ over all $a_{i}, a_{j}, b_{i}, b_{j}$, except $\infty$, covers each nonzero element of $G$ an equal number of times.

Then a cyclic development of this set of $n / 2$ pairs (which defines a board) yields a $\operatorname{BHR}(n)$, with requirement (R1) guaranteeing condition (i) and requirement (R2) guaranteeing condition (ii), since the cyclic development preserves differences.

By letting $\left\{a_{1}, a_{2}, \ldots, a_{n / 2}\right\}=C_{0}+U+\{\infty\}, \quad\left\{b_{1}, b_{2}, \ldots, b_{n / 2}\right\}=C_{1}+V-$ $\{0\}$, requirement $(\mathrm{R} 2)$ is automatically satisfied. It suffices to produce a pairing between $\left\{a_{i}\right\}$ and $\left\{b_{j}\right\}$ which satisfies requirement (R1). We first prove some lemmas.

Lemma 1. Suppose that $j, k, l, m$ satisfy the conditions

$$
x^{2 k}+x^{j}=x^{m}, \quad 0 \leqslant m-j \leqslant P-2, \quad-2 y^{j+2 l}=1 .
$$

Furthermore, suppose that (i) when $0 \leqslant m-j \leqslant(P-1) / 2$, then $2 j+2 l-$ $m-(P+1) / 2$ is either 0 or 1 , (ii) when $(P-1) / 2 \leqslant m-j \leqslant P-2$, then $2 j+2 l$ $-m-(P+1) / 2$ is either 1 or 2 . Then there exists a pairing satisfying requirements (R1) and (R2).

Proof. We demonstrate pairings between elements in $C_{0}+U+\{\infty\}$ and elements in $C_{1}+V-\{0\}$ satisfying requirement (R1) for both case (i) and case (ii). 
Case (i). The pairing is:

(1)

$$
\left(x^{i+2 k}, y^{i}\right) \text { vs. }\left(-x^{i+j},-y^{i+j+2 l}\right), \quad(P-1) / 2 \leqslant i \leqslant d-1,
$$

(2) $\left(x^{i+2 k}, y^{i}\right)$ vs. $\left(0, y^{i}\right), \quad 0 \leqslant i \leqslant(P-3) / 2$,

$$
\left(-x^{i+j}, 0\right) \text { vs. }\left(-x^{i+j},-y^{i+j+2 l}\right), \quad 0 \leqslant i \$(P-3) / 2,
$$

$$
\left(-x^{i+j}, 0\right) \text { vs. }\left(0, y^{i+2 j+2 l-m-(P+1) / 2}\right) \text {, }
$$

$$
\begin{array}{r}
(P-1) / 2 \leqslant i \leqslant m+(P-3) / 2-j, \\
\left(-x^{i+j}, 0\right) \text { vs. }\left(0, y^{i+2 j+2 l-m-(P+1) / 2+1}\right), \\
m+(P-1) / 2-j \leqslant i \leqslant P-2,
\end{array}
$$
$(0,0)$ vs. $\left(0, y^{j+2 l-1}\right)$,

$$
\begin{array}{ll}
\infty \text { vs. }\left(0, y^{P}\right), & \text { if } 2 j+2 l-m-(P+1) / 2=0, \\
\infty \text { vs. }\left(0, y^{(P-1) / 2}\right), & \text { if } 2 j+2 l-m-(P+1) / 2=1 .
\end{array}
$$

The symmetric differences are:

$$
\begin{array}{cc} 
\pm\left(x^{i+m},-y^{i+j+2 l}\right), & (P-1) / 2 \leqslant i \leqslant d-1, \\
\pm\left(x^{i+2 k}, 0\right), & 0 \leqslant i \leqslant(P-3) / 2, \\
\pm\left(0, y^{i+j+2 l}\right), & 0 \leqslant i \leqslant(P-3) / 2, \\
\pm\left(x^{i+j}, y^{i+2 j+2 l-m-(P+1) / 2}\right), & (P-1) / 2 \leqslant i \leqslant m+(P-3) / 2-j, \\
= \pm\left(x^{i+m}, y^{i+j+2 l-(P+1) / 2}\right), & (P-1) / 2-m+j \leqslant i \leqslant(P-3) / 2, \\
= \pm\left(x^{i+m},-y^{i+j+2 l}\right), \quad(P-1) / 2-m+j \leqslant i \leqslant(P-3) / 2, \\
\pm\left(x^{i+j}, y^{i+2 j+2 l-m-(P+1) / 2+1}\right), \quad m+(P-1) / 2-j \leqslant i \leqslant P-2, \\
= \pm\left(x^{i+m-(P-1) / 2}, y^{i+j+2 l}\right), \quad 0 \leqslant i \leqslant(P-3) / 2-m+j, \\
= \pm\left(x^{i+m},-y^{i+j+2 l}\right), \quad 0 \leqslant i \leqslant(P-3) / 2-m+j, \\
\pm\left(0, y^{j+2 l-1}\right)=\left(0,-y^{(P-1) / 2+j+2 l}\right)= \pm\left(0, y^{(P-1) / 2+j+2 l}\right) .
\end{array}
$$

Case (ii). The pairing is:

(1) $\left(x^{i+2 k}, y^{i}\right)$ vs. $\left(-x^{i+j}, y^{i+j+2 l}\right), \quad(P-1) / 2 \leqslant i \leqslant d$,

(2) $\quad\left(x^{i+2 k}, y^{i}\right)$ vs. $\left(0, y^{i}\right), \quad 0 \leqslant i \leqslant(P-3) / 2$,

(3) $\quad\left(-x^{i+j}, 0\right)$ vs. $\left(-x^{i+j}, y^{i+j+2 l}\right), \quad 0 \leqslant i \leqslant(P-3) / 2$,

(4) $\quad\left(-x^{i+j}, 0\right)$ vs. $\left(0, y^{i+2 j+2 l-m-(P+1) / 2-1}\right), \quad(P-1) / 2 \leqslant i \leqslant m-j-1$,

(5) $\quad\left(-x^{i+j}, 0\right)$ vs. $\left(0, y^{i+2 j+2 l-m-(P+1) / 2}\right), \quad m-j \leqslant i \leqslant P-2$,

(6) $\quad(0,0)$ vs. $\left(0, y^{j+2 l-(P+3) / 2}\right)$, $\infty$ vs. $\left(0, y^{P}\right), \quad$ if $2 j+2 l-m-(P+1) / 2=1$, $\infty$ vs. $\left(0, y^{(P-1) / 2}\right) \quad$ if $2 j+2 l-m-(P+1) / 2=2$. 
The symmetric differences are:

$$
\begin{gathered}
\pm\left(x^{i+m}, y^{i+j+2 l}\right), \quad(P-1) / 2 \leqslant i \leqslant d-1, \\
\pm\left(x^{i+2 k}, 0\right), \quad 0 \leqslant i \leqslant(P-3) / 2, \\
\pm\left(0, y^{i+j+2 l}\right), \quad 0 \leqslant i \leqslant(P-3) / 2, \\
\pm\left(x^{i+j}, y^{i+2 j+2 l-m-(P+1) / 2-1}\right), \quad(P-1) / 2 \leqslant i \leqslant m-j-1, \\
= \pm\left(x^{i+j}, y^{i+2 j+2 l-m+(P+1) / 2-1}\right), \quad(P-1) / 2 \leqslant i \leqslant m-j-1, \\
= \pm\left(x^{i+m-(P-1) / 2}, y^{i+j+2 l}\right), \quad P-1-m+j \leqslant i \leqslant(P-3) / 2, \\
= \pm\left(x^{i+m},-y^{i+j+2 l}\right), \quad P-1-m+j \leqslant i \leqslant(P-3) / 2, \\
\pm\left(x^{i+j}, y^{i+2 j+2 l-m-(P+1) / 2}\right), \quad m-j \leqslant i \leqslant P-2, \\
= \pm\left(x^{i+m}, y^{i+j+2 l-(P+1) / 2}\right), \quad 0 \leqslant i \leqslant P-2-m+j, \\
= \pm\left(x^{i+m},-y^{i+j+2 l}\right), \quad 0 \leqslant i \leqslant P-2-m+j, \\
\pm\left(0, y^{j+2 l-(P+3) / 2}\right)= \pm\left(0, y^{(P-1) / 2+j+2 l}\right) .
\end{gathered}
$$

In both cases, it is straightforward to verify that the pairings and the symmetric differences are indeed what we want. Note that if $m-j=(P-1) / 2$, then subcases (i)(5) and (ii)(4) do not occur.

Lemma 2. Suppose that $k, m, z$ satisfy the following conditions:

$$
x^{2 k}+1=x^{m}, \quad 0 \leqslant m \leqslant P-2, \quad 2=y^{z} .
$$

Furthermore, suppose that (i) when $0 \leqslant m \leqslant(P-1) / 2$, then $z-m$ is either 0 or 1 , (ii) when $(P-1) / 2 \leqslant m \leqslant P-2$, then $z-m$ is either 1 or 2 . Then there exists $a$ pairing satisfying requirements $(\mathrm{R} 1)$ and $(\mathrm{R} 2)$.

Proof. Case (i). The pairing is:

$$
\begin{aligned}
& \left(x^{i+2 k}, y^{i}\right) \text { vs. }\left(-x^{i},-y^{i}\right), \quad(P-1) / 2 \leqslant i \leqslant d-1, \\
& \left(x^{i+2 k}, y^{i}\right) \text { vs. }\left(0, y^{i}\right), \quad 0 \leqslant i \leqslant(P-3) / 2 \text {, } \\
& \left(-x^{i}, 0\right) \text { vs. }\left(-x^{i},-y^{i}\right), \quad 0 \leqslant i \leqslant(P-3) / 2, \\
& \left(-x^{i}, 0\right) \text { vs. }\left(0, y^{i+z-m}\right), \quad(P-1) / 2 \leqslant i \leqslant(P-3) / 2+m, \\
& \left(-x^{i}, 0\right) \text { vs. }\left(0, y^{i+z-m+1}\right), \quad(P-1) / 2+m \leqslant i \leqslant P-2 \text {, } \\
& (0,0) \mathrm{v} s .\left(0, y^{P}\right), \quad \text { if } z-m=0, \\
& (0,0) \text { vs. }\left(0, y^{(P-1) / 2}\right), \quad \text { if } z-m=1 \text {, } \\
& \infty \text { vs. }\left(0, y^{z+(P-1) / 2}\right) \text {. }
\end{aligned}
$$

The symmetric differences are:

$$
\begin{array}{cc} 
\pm\left(x^{i+m}, y^{i+z}\right), & (P-1) / 2 \leqslant i \leqslant d-1, \\
\pm\left(x^{i+2 k}, 0\right), & 0 \leqslant i \leqslant(P-3) / 2, \\
\pm\left(0, y^{i}\right), & 0 \leqslant i \leqslant(P-3) / 2,
\end{array}
$$


(4)

$$
\begin{gathered}
\pm\left(x^{i}, y^{i+z-m}\right), \quad(P-1) / 2 \leqslant i \leqslant(P-3) / 2+m, \\
= \pm\left(x^{i+m}, y^{i+z}\right), \quad(P-1) / 2-m \leqslant i \leqslant(P-3) / 2 \\
\pm\left(x^{i}, y^{i+z-m+1}\right), \quad(P-1) / 2+m \leqslant i \leqslant P-2 \\
= \pm\left(x^{i+m}, y^{i+z+1}\right), \quad(P-1) / 2 \leqslant i \leqslant P-2-m \\
= \pm\left(x^{i+m}, y^{i+z}\right), \quad 0 \leqslant i \leqslant(P-3) / 2-m, \\
\pm\left(0, y^{P}\right) \pm\left(0, y^{P}\right)= \pm\left(0, y^{(P-1) / 2}\right), \quad \text { if } z-m=0 \\
\pm\left(0, y^{(P-1) / 2}\right), \quad \text { if } z-m=1 .
\end{gathered}
$$

Case (ii). The pairing is:

$$
\begin{aligned}
& \left(x^{i+2 k}, y^{i}\right) \text { vs. }\left(-x^{i},-y^{i}\right), \quad(P-1) / 2 \leqslant i \leqslant d-1, \\
& \left(x^{i+2 k}, y^{i}\right) \text { vs. }\left(0, y^{i}\right), \quad 0 \leqslant i \leqslant(P-3) / 2,
\end{aligned}
$$$$
\left(-x^{i}, 0\right) \text { vs. }\left(-x^{i},-y^{i}\right), \quad 0 \leqslant i \leqslant(P-3) / 2,
$$$$
\left(-x^{i}, 0\right) \text { vs. }\left(0, y^{i+z-m-1}\right), \quad(P-1) / 2 \leqslant i \leqslant m-1,
$$$$
\left(-x^{i}, 0\right) \text { vs. }\left(0, y^{i+z-m}\right), \quad m \leqslant i \leqslant P-2 \text {, }
$$

$$
\begin{array}{ll}
(0,0) \text { vs. }\left(0, y^{P}\right), & \text { if } z-m=1, \\
(0,0) \text { vs. }\left(0, y^{(P-1) / 2}\right), & \text { if } z-m=2,
\end{array}
$$

The symmetric differences are:

(1)

$$
\begin{array}{cc} 
\pm\left(x^{i+m}, y^{i+z}\right), & (P-1) / 2 \leqslant i \leqslant d-1 \\
\pm\left(x^{i+2 k}, 0\right), & 0 \leqslant i \leqslant(P-3) / 2, \\
\pm\left(0, y^{i}\right), & 0 \leqslant i \leqslant(P-3) / 2, \\
\pm\left(x^{i}, y^{i+z-m-1}\right), & (P-1) / 2 \leqslant i \leqslant m-1,
\end{array}
$$

$$
\begin{gathered}
= \pm\left(x^{i}, y^{i+z-m+P}\right), \quad(P-1) / 2 \leqslant i \leqslant m-1, \\
= \pm\left(x^{i+m-(P-1) / 2}, y^{i+z-(P+1) / 2}\right), \quad P-1-m \leqslant i \leqslant(P-3) / 2, \\
= \pm\left(x^{i+m}, y^{i+z}\right), \quad P-1-m \leqslant i \leqslant(P-3) / 2, \\
\quad \pm\left(x^{i}, y^{i+z-m}\right), \quad m \leqslant i \leqslant P-2, \\
= \pm\left(x^{i+m}, y^{i+z}\right), \quad 0 \leqslant i \leqslant P-2-m, \\
\pm\left(0, y^{P}\right)=\left(0, y^{(P-1) / 2}\right), \quad \text { if } z-m=1, \\
\pm\left(0, y^{(P-1) / 2}\right), \quad \text { if } z-m=2 .
\end{gathered}
$$

Note that when $m=(P-1) / 2$, then subcases (i)(5) and (ii)(4) do not occur.

3. Proof of Theorem 2. Let $x$ be a generator of $\mathrm{GF}^{*}(P)$. For $u \in \mathrm{GF}^{*}(P)$, define $\log _{x} u=i$ if $u=x^{i}, 0 \leqslant i \leqslant P-2$. Similarly, we can define $\log _{y} v$ for $v \in \mathrm{GF}^{*}(Q)$. Let $\log _{y} 2=z$. Then $z \neq(P+1) / 2$ since $2=y^{z}=y^{(P+1) / 2}=-1$ implies $q=3$, a contradiction to our assumption. We consider four other possible cases. 
Case (i). $1 \leqslant z \leqslant(P-1) / 2, \log _{x}\left(x^{z}-1\right) \equiv 1(\bmod 2)$.

Set $j=0$ or 1 where $j \equiv(P+1) / 2-z(\bmod 2)$,

$$
\begin{gathered}
2 l=3(P+1) / 2-z-j, \quad 2 k=2 j+2 l-3+\log _{x}\left(x^{z}-1\right), \\
m=2 j+2 l-(P+1) / 2-2 .
\end{gathered}
$$

We now verify that the conditions in Lemma 1 (ii) are satisfied.

First of all it is easily seen that both $2 l$ and $2 k$ are even. So $k$ and $l$ are well defined. Furthermore

$$
\begin{aligned}
x^{2 k}+x^{j} & =x^{2 j+2 l-3+\log _{x}\left(x^{z}-1\right)}+x^{j} \\
& =x^{m+(P-1) / 2}\left(x^{z}-1\right)+x^{j}=-x^{m}\left(x^{3(P+1) / 2-j-2 l}-1\right)+x^{j} \\
& =-x^{m}\left(x^{(P+1) / 2-j-2 l+2}-1\right)+x^{j}=-x^{j}+x^{m}+x^{j}=x^{m}, \\
-2 y^{j+2 l} & =-2 y^{3(P+1) / 2-z}=-2(-1)\left(\frac{1}{2}\right)=1 .
\end{aligned}
$$

Finally,

$$
2 j+2 l-m-(P+1) / 2=2,
$$

and

$$
m-j=j+2 l-(P+1) / 2-2=P+1-z-2=P-1-z
$$

imply $(P-1) / 2 \leqslant m-j \leqslant P-2$. Thus Theorem 2 follows from Lemma 1(ii).

Case (ii). $1 \leqslant z \leqslant(P-1) / 2, \log _{x}\left(x^{z}-1\right) \equiv 0(\bmod 2)$.

Set $m=z, 2 k=\log _{x}\left(x^{z}-1\right)$. We now verify that the conditions in Lemma 2(i) are satisfied. Clearly, $2 k$ is even. Furthermore

$$
x^{2 k}+1=x^{z}-1+1=x^{m} .
$$

Finally, by our assumptions,

$$
y^{z}=2, \quad 0 \leqslant m \leqslant(P-1) / 2,
$$

and $z-m=0$.

Case (iii). $(P+3) / 2 \leqslant z \leqslant P, \log _{x}\left(x^{z-2}-1\right) \equiv 1(\bmod 2)$.

Set $j=0$ or 1 where $j \equiv(P+1) / 2-z(\bmod 2)$,

$$
\begin{gathered}
2 l=3(P+1) / 2-z-j, \quad 2 k=2 j+2 l-1+\log _{x}\left(x^{z-2}-1\right), \\
m=2 j+2 l-(P+1) / 2 .
\end{gathered}
$$

The verification that the conditions in Lemma 1(i) are satisfied is similar to case (i).

Case (iv). $(P+3) / 2 \leqslant z \leqslant P, \log _{x}\left(x^{z-2}-1\right) \equiv 0(\bmod 2)$.

Set $m=z-2,2 k=\log _{x}\left(x^{z-2}-1\right)$.

The verification that the conditions in Lemma 2(ii) are satisfied is similar to case (ii). The proof is complete.

\section{Examples.}

ExAmple 1. $n=16, P=3, Q=5, d=4$.

$x=2$ and $y=2$ are generators of $\mathrm{GF}^{*}(3)$ and $\mathrm{GF}^{*}(5)$, respectively. Since $z=\log _{y} 2=1$ and $\log _{x}\left(x^{3}-1\right) \equiv 0(\bmod 2)$, we set

$$
m=z=1, \quad 2 k=\log _{x}\left(x^{z}-1\right)=2,
$$


and use the pairing of Lemma 2(i), i.e.,

$$
\begin{aligned}
& (2,2) \text { vs. }(1,3), \\
& (1,4) \text { vs. }(2,1), \\
& (2,3) \text { vs. }(1,2), \\
& (1,1) \text { vs. }(0,1), \\
& (2,0) \text { vs. }(2,4), \\
& (1,0) \text { vs. }(0,2), \\
& (0,0) \text { vs. }(0,3), \\
& \infty \text { vs. }(0,4) .
\end{aligned}
$$

EXAmple 2. $n=36, P=5, Q=7, d=12$.

$x=2$ and $y=3$ are generators of $\mathrm{GF}^{*}(5)$ and $\mathrm{GF}^{*}(7)$, respectively. Since $z=\log _{y} 2=2$ and $\log _{x}\left(x^{z}-1\right) \equiv 1(\bmod 2)$, we set

$$
\begin{gathered}
j=1 \equiv(P+1) / 2-z(\bmod 2), \quad 2 l=3(P+1) / 2-z-j=6, \\
2 k=2 j+2 l-3+\log _{x}\left(x^{z}-1\right)=8, \quad m=2 j+2 l-(P+1) / 2-2=3,
\end{gathered}
$$

and use the pairing of Lemma 1(ii), i.e.,

$\begin{array}{llll}(1) & (4,2) \text { vs. }(2,1), & (3,6) \text { vs. }(4,3), & (1,4) \text { vs. }(3,2), \\ & (2,5) \text { vs. }(1,6), & (4,1) \text { vs. }(2,4), & (3,3) \text { vs. }(4,5), \\ & (1,2) \text { vs. }(3,1), & (2,6) \text { vs. }(1,3), & \\ & (4,4) \text { vs. }(2,2), & (3,5) \text { vs. }(4,6), \\ (2) & (1,1) \text { vs. }(0,1), & (2,3) \text { vs. }(0,3), \\ (3) & (3,0) \text { vs. }(3,4), & (1,0) \text { vs. }(1,5), \\ (5) & (2,0) \text { vs. }(0,4), & (4,0) \text { vs. }(0,5), \\ (6) & (0,0) \text { vs. }(0,6), & \\ (7) & \infty & \text { vs. }(0,2) .\end{array}$

\section{REFERENCES}

1. E. R. Berlekamp and F. K. Hwang, Constructions for balanced Howell rotations for bridge tournaments, J. Combin. Theory Ser. A 12 (1972), 159-166.

2. D.-Z. Du and F. K. Hwang, Symmetrical skew balanced starters and complete balanced Howell rotations, Trans. Amer. Math. Soc. 271 (1982), 409-413.

3. F. K. Hwang, New constructions for balanced Howell rotations, J. Combin. Theory Ser. A 21 (1976), 44-51.

4. F. K. Hwang, Q. D. Kang and J. E. Yu, Complete balanced Howell rotations for $16 k+12$ teams (to appear).

5. E. T. Parker and A. N. Mood, Some balanced Howell rotations for duplicate bridge sessions, Amer. Math. Monthly 62 (1955), 714-716.

6. P. J. Schellenberg, Constructions for (balanced) Room squares, Aequationes Math. 9 (1973), 75-90.

7. T. Storer, Cyclotomy and difference sets, Markham, Chicago, Ill., 1967.

Institute of Applied Mathematics, ACAdemy of Science, Beijing, China

Bell laboratories, Murray Hill, New Jersey 07974 